\title{
Fiabilidad y validez de la escala de actitudes hacia el medio ambiente natural para adolescentes (Aman-a)
}

Daniel Musitu-Ferrer

Universidad Pablo de Olavide (España)

Macarena Esteban Ibáñez

Universidad Pablo de Olavide (España)
Juan Evaristo Callejas Jerónimo

Universidad Pablo de Olavide (España)

Luis V. Amador-Muñoz

Universidad Pablo de Olavide (España)

Celeste León-Moreno

Universidad Pablo de Olavide (España) 



\section{Fiabilidad y validez de la escala de actitudes hacia el medio ambiente natural para adolescentes (Aman-a)}

\section{Reliability and validity of the adolescents's attitudes towards natural environment scale (Aman-a)}

\author{
Daniel Musitu-Ferrer \\ Universidad Pablo de Olavide (España) \\ dmusfer@alu.upo.es
}

\section{Macarena Esteban Ibáñez}

Universidad Pablo de Olavide (España)

mestiban@upo.es

\section{Celeste León-Moreno}

Universidad Pablo de Olavide (España)

cmleomor@upo.es
Juan Evaristo Callejas Jerónimo

Universidad Pablo de Olavide (España)

jevaristocallejas@gmail.com

\section{Luis V. Amador-Muñoz}

Universidad Pablo de Olavide (España)

lvamador@upo.es

Fecha de recepción: 1 de septiembre de 2019

Fecha de aceptación: 23 de enero de 2020

\section{Resumen}

El objetivo de este trabajo fue construir y validar una escala de actitudes hacia el medio ambiente natural en adolescentes españoles. Se generaron 72 ítems a partir de los escritos de 264 adolescentes sobre actitudes hacia el medio ambiente. A través del juicio de expertos y de un estudio piloto se efectuó una depuración reduciendo la escala a 9 ítems que se administraron a una muestra de 833 adolescentes de ambos sexos. Mediante un análisis factorial exploratorio, se identificaron dos dimensiones, actitud proximal y actitud distal, que fueron replicadas a través de un análisis confirmatorio cuyos resultados mostraron un buen ajuste a los datos. Los índices de fiabilidad fueron adecuados y respecto de la validez se obtuvieron relaciones significativas con la empatía con el medio ambiente natural en sus dos dimensiones, cognitiva y emocional, y el ajuste escolar. También se obtuvieron diferencias significativas en función del género en la actitud distal, pero no en la actitud proximal. Se analizan los resultados obtenidos y se subraya la importancia de esta escala en la investigación ambiental en la adolescencia.

Palabras clave: Actitudes hacia el medio ambiente; Empatía con el medio ambiente; Ajuste escolar; Adolescencia 


\begin{abstract}
The aim of this study was to design and validate a scale of attitudes towards the natural environment among Spanish teenagers. 72 items were generated from the writings of 264 adolescents on attitudes towards the environment. Through expert judgement and a pilot study, a depuration was carried out, reducing the scale to 9 items that were administered to a sample of 833 adolescents of both sexes. By means of an exploratory Factor analysis, two dimensions were identified, proximal attitude and distal attitude, which were replicated through a confirmatory analysis whose results showed a good fit to the data. Reliability indices were adequate and with respect to validity significant relationships were obtained with empathy with the natural environment in its two dimensions, cognitive and emotional, and school adjustment. Significant gender differences were also found in distal, but not proximal, attitudes. Results obtained are analysed and the importance of this scale in environmental research in adolescence is also highlighted.
\end{abstract}

Keywords: Attitudes towards the environment; Empathy with the environment; School adjustment; Adolescence

\title{
1.INTRODUCCIÓN
}

En estas últimas décadas, se han llevado a cabo numerosas investigaciones para responder a los problemas ambientales que nos aquejan, promoviendo un cambio de actitud respecto de las relaciones entre los seres humanos y la naturaleza y considerando que la educación es una disciplina fundamental para difundir los principios del desarrollo sostenible (Biasutti y Frate, 2017; Esteban Ibáñez, Amador Muñoz y Mateos Claros, 2017; Heberlein, 2012; Levine y Strube, 2012; Michalos et al., 2012; Olsson, Gericke y Rundgren, 2016; Tilbury, 2012).

Los problemas ambientales en nuestros días tales como la contaminación de los mares, la disminución de la capa de ozono, el calentamiento global, la destrucción de los bosques y de otros recursos naturales, el cambio climático, etc... han ido calando, aunque muy lentamente, en la ciudadanía en forma de cambio de actitud, más favorable hacia el medio ambiente, y de cambio de conducta más pro-ambiental y sustentable (Akehurst, Afonso y Gonçalves, 2012, Duarte, Escario y Sanagustín, 2017; Kotler, Kartajava y Setiawan, 2011; Miguens, Álvarez, González y García, 2015).

En este escenario, la educación ambiental se enfrenta según Novo (2009) a dos retos de gran calado si realmente se desea lograr un mundo sostenible: 1) el reto ecológico que supone formar y educar no solo a niños y jóvenes, sino también a todos los implicados en la preservación del medio ambiente natural, de manera que se logre la armonía entre el ser humano y la naturaleza; para lograr esta armonía se requiere de un fortalecimiento y, en ocasiones, de un cambio profundo de las actitudes y valores respecto del medio ambiente natural; y, 2), el reto de la transformación y del cambio social para lograr una mayor igualdad y equilibrio social de manera que los recursos naturales no sean los paganos de las profundas desigualdades sociales. De ahí que la educación ambiental se considere como un proceso que pretende formar y crear 
conciencia en los seres humanos hacia su entorno, generando en ellos un grado de compromiso, responsabilidad y un modo de vida compatible con la sustentabilidad, mediante la adecuada exploración, explotación, utilización, y manejo de los recursos naturales (Amador Muñoz y Esteban Ibáñez, 2011; Ariza, 2017; Stevenson, Peterson y Bondell, 2016).

La educación ambiental tiene como objetivo fundamental formar ciudadanos comprometidos con la preservación del medio ambiente natural, de manera que puedan comprender e interiorizar su relación y recíproca dependencia (Ariza, 2017; Vargas, Moreno, Vázquez y Gutiérrez, 2011). También, en la educación ambiental se encuentra implícita la idea de que los sistemas de actitudes y creencias que tenemos respecto del medio ambiente natural son la raíz de los problemas ambientales y de su solución (Heyl, Díaz y Cifuentes, 2013; Moyano-Díaz, Cornejo y Gallardo, 2011). Son numerosos los trabajos en los que se constata que las conductas proambientales se predicen a partir de factores internos y externos (Bamberg y Moser, 2007; Heyl et al., 2013; Kollumuss y Agyeman, 2002; Moyano-Díaz et al., 2011) y entre los primeros, uno de los más relevantes es la actitud hacia el medio ambiente natural (CorralVerdugo, Frijo y Tapia, 2004; Kaiser, 1998, Makki, Abd-el-khalick y Boujaoude, 2003; Moyano-Díaz et al., 2011). Por tanto, parece imprescindible considerar la educación ambiental como un gran recurso para el beneficio óptimo de las relaciones del ser humano con su medio, a través del conocimiento, sensibilización, promoción de estilos de vida saludables y comportamientos proambientales.

\subsection{Actitudes y medio ambiente natural}

A lo largo del tiempo han sido numerosas las formas de acercarse al término actitud. Siempre se ha considerado como una forma de posicionarse ante algo, como una tendencia, como una predisposición mental o física o bien como una respuesta de carácter evaluador y relativamente estable (Allport, 1968; Katz y Scotland, 1959; Lamberth, 1980). Dicho de otra forma, se podría considerar como una posición que expresa un estado de ánimo o una intención, o, una forma de ver el mundo. El propio Allport (1968, p. 45) las concibe como: "Una predisposición mental y neuronal que se adquiere a través de la experiencia, que ejerce una influencia o dinámica en la respuesta del individuo a toda clase de objetos o situaciones con las que se relaciona". En el ámbito de la educación ambiental, Holahan (1982, p. 15), las definió como: "los sentimientos favorables o desfavorables que se tiene hacia alguna característica del medio ambiente natural o hacia un problema relacionado con él"; y, Taylord y Todd (1995), la definieron como un determinante directo de la predisposición hacia acciones a favor del medio ambiente natural.

Por tanto, la actitud se considera como un constructo mediador que intercede entre los estímulos del ambiente social de la persona y las respuestas y reacciones de ésta a dichos estímulos. La actitud, en relación con nuestros valores, sería desde 
este punto de vista, una forma de adaptación activa de los individuos a su medio ambiente. Es activa por tratarse del resultado de las experiencias del individuo con el objeto actitudinal y, por tanto, de las conclusiones de los procesos cognitivos, afectivos y conductuales manifestados en dichas relaciones o experiencias (Melero y Buz, 2002).

Las actitudes no son innatas, se aprenden a partir de una sucesión de experiencias con las que se refuerzan o no determinadas conductas. De ahí que supongan una predisposición a la acción relativamente estable, siendo susceptibles de formación y cambio, a la vez que son relativamente duraderas, entre otras, por las siguientes razones: 1) las afinidades y aversiones que expresamos hacia los objetos o cosas están arraigadas en nuestras emociones. Ambas, especialmente las segundas, son difíciles de extinguir, lo que facilita su duración; y, 2) los sentimientos no se pueden separar de nuestras percepciones, en la medida en que dirigen nuestra atención y percepción a determinados aspectos de la realidad (Escalante, Repetto y Mattinello, 2012).

En consecuencia, el medio ambiente en el que está integrado el sujeto reforzará sus propias actitudes (Olsson et al., 2016; Pérez Serrano, 2002). Un aspecto esencial de las actitudes es el que hace referencia a su estructura. Normalmente se han asumido dos orientaciones, en parte complementarias: el enfoque clásico (Rosenberg y Hovland, 1960) que hace referencia a los componentes que lo conforman: cognitivo -las actitudes están conformadas por conjuntos estructurados de creencias, valores, conocimientos o expectativas, relativamente estables que predisponen a actuar de un modo preferente ante un objeto o situación -son las opiniones, creencias, categorías, atributos y conceptos.-; afectivo - es el elemento de mayor importancia en la configuración de las actitudes (Fishbein y Ajzen, 1977) y está conformado por los sentimientos hacia el objeto de la actitud - es la valoración que determina la conducta y se activa motivacionalmente ante la presencia del objeto o situación, viniendo acompañada de sentimientos-; $y$, finalmente, el conativo comportamental) que hace referencia a la predisposición o la intención a actuar y se manifiesta orientando la acción de una manera determinada ante un objeto o sujeto (Eagly y Chaiken, 1993; Lamberth, 1980). La segunda orientación hace referencia al modelo de Fishbein y Ajzen (1977) en el que sus componentes se incrementan a cuatro: el cognitivo (las creencias y opiniones), el afectivo (sentimientos y evaluación), el conativo (las intenciones conductuales) y la conducta (conducta observada a través de los actos) y al que denominaron modelo de valor-expectativa. Posteriormente, con algunas modificaciones, dio lugar a la teoría de la acción razonada (Fishbein y Ajzen, 1980) y, finalmente, a la teoría de la acción planificada (Ajzen y Madden, 1986). Estos modelos lo que hacen es ilustrar el rol que desempeñan las actitudes en la formación de las conductas, al considerar que para cambiar las conductas en un ámbito específico es indispensable modificar las actitudes. 


\subsection{Medida de las actitudes}

En la última década se han desarrollado numerosas escalas y cuestionarios con la finalidad de evaluar las actitudes hacia el medio ambiente natural (Fraijo, Tapia, y Corral, 2007; Michalos et al., 2012; Olsson et al., 2016; Powell, Stern, Krohn y Ardoin, 2011; Schneller, Johnson y Bogner, 2015). La idea subyacente es siempre que las actitudes positivas respecto del medio ambiente natural son antecedentes importantes del comportamiento proambiental (Brody, Highfield y Alston, 2004; Fielding, Terry, Masser y Hogg, 2008; Foster y Mcbeth, 1996; Harris, 2006; Raudsepp, 2001; Schultz, 2000). Por esta razón, y como decía Milfont y Duckitt (2004), un adecuado examen y comprensión de las actitudes ambientales de la ciudadanía es, probablemente, una vía importante para promover las conductas proambientales.

El problema con el que nos encontramos en el momento actual es que no existe una verdadera convergencia respecto del uso de una escala común que mida las actitudes respecto del medio ambiente natural y respecto del desarrollo sustentable, $\mathrm{y}$, paralelamente, tampoco una definición compartida por los investigadores, lo cual es atribuible a los diferentes antecedentes teóricos que subyacen en la construcción de las escalas y, consecuentemente, en la definición de actitud (Schneller et al., 2015). Serían de interés subrayar dos modelos que han tenido un gran impacto en la construcción de las escalas de actitudes hacia el medio ambiente natural: el Modelo de Valores Ecológicos (2-MEV) (Bogner y Wiseman, 2006; Schneller et al., 2015); y el nuevo paradigma ambiental (NEP) que evalúa con carácter general las creencias acerca de la tierra y la biosfera y las repercusiones que tiene la conducta humana en su equilibrio (Dunlap, Van Liere, Mertig y Jones, 2000; Fleury-Bahi, Marcouyeux, Renard y Roussiau, 2015; Harris, 2006; Shephard et al., 2014).

Y, en este mismo periodo, aunque con distinta orientación y también más específica, es de destacar la escala de Preocupación Ambiental de Weigel y Weigel (1978) en el ámbito anglosajón, que evalúa a gran escala los aspectos relacionados con la conservación y la contaminación de la naturaleza y, la de Castadeno (1995), en el ambito español, que evalua las actitudes pro-ambientales en alumnos universitarios.

En general, estos modelos han sido, en muchos casos, el fundamento teórico y la fuente de inspiración para la construcción de diferentes medidas en el ámbito de la educación ambiental, como por ejemplo la escala de actitudes hacia el medio ambiente natural del International Social Survey Program cuya finalidad fue evaluar la preocupación por el medio ambiente natural en 20 países obteniendo un índice global de preocupación por el medio ambiente (ISSP,1993). También es interesante subrayar la encuesta de Salud del Planeta (Dunlap, Gallup, y Gallup, 1993) cuya 
finalidad fue la de obtener información respecto del medio ambiente, la extinción de las especies, la contaminación, la tala de los bosques, etc... (Moreno, Corraliza y Ruiz, 2005) y la escala de actitudes hacia el medio ambiente natural (Milfont y Duckitt, 2010).

En el ámbito de la infancia también se han elaborado interesantes escalas para evaluar actitudes como, por ejemplo, la escala de actitudes y conocimientos ambientales para niños (Leeming, Dwyer y Bracken, 1995); las escalas para evaluar las actitudes y conductas hacia el medio ambiente natural de niños de seis y siete años (Evans et al., 2017); la escala para evaluar a niños de 8 a 12 años del sur de Brasil (Galli, de Campos, Bedin y Sarrieta, 2013); la adaptación y prueba de una escala de orientación hacia la sustentabilidad en niños de sexto año de educación básica (Fraijo, Corral, Tapia y García, 2012); el Inventario de Reacción Ambiental de ocho ítems para medir las actitudes ambientales de los niños en edad preescolar de Kesícíoğlu y Alísínanoğlu (2009) y la escala de Soydan y Samur (2014) para evaluar las actitudes ambientales de los niños en edad preescolar y sus madres a través de variables como el sexo, la edad y el nivel educativo (Kroufek et al., 2016). Y, finalmente, también se han elaborado escalas en el ámbito de la adolescencia, como la valoración del cambio de actitudes hacia el medio ambiente producido por el programa didáctico EICEA (Vega, Ferra y Quijano, 2009); la evaluación de actitudes de alumnos de la ESO (Álvarez, Inmaculada de la Fuente, García y Fernández, 2009); la escala de actitudes, conciencia e intención conductual del alumnado de diez a dieciséis años (Bergman, 2016); las escalas de actitudes y conductas respecto del desarrollo sustentable que se fundamentan en varios documentos de la UNESCO (Biasutti y Surian, 2012; Michalos et al., 2012; Ogunbode, 2013; Olsson et al., 2016); la escala para evaluar la Actitud hacia el Cambio Climático a partir de las Creencias e Intenciones de los Estudiantes de Secundaria para Promulgar un Cambio Ambiental Positivo (Christensen y Knezek, 2015) y más recientemente la escala de actitud ambiental (EAS en inglés) para adolescentes de Uzun et al., (2019).

Sin embargo, y considerando la calidad de las escalas realizadas hasta el momento presente, se han constatado dos aspectos que consideramos importante resaltar: 1) son muy pocas las escalas realizadas en el contexto de habla hispana cuyo objetivo sea estudiar las actitudes de los adolescentes hacia el medio ambiente natural y, 2) en la construcción de las escalas realizadas en el ámbito internacional, al menos hasta donde hemos podido explorar, no se realizan desde la perspectiva de los adolescentes, lo cual lleva implícito el riesgo de los sesgos en las respuestas debido a que se les pregunta sobre aspectos sobre los que nunca han pensado. De alguna manera de lo que se trata con este artículo es construir una escala que represente el pensar y sentir de los adolescentes. Por ello, el propósito de la presente investigación fue construir y validar una escala de actitudes hacia el medio ambiente natural desde la perspectiva de los adolescentes. 


\section{MÉTODO}

\subsection{Participantes}

La muestra estaba constituida por 881 adolescentes de la que fueron excluidos 48 por las siguientes razones: $72 \%$ por no responder algunas de las escalas o responder de la misma manera a una o más escalas; $20 \%$ por problemas de compresión y $8 \%$ abandonaron el estudio. La muestra final fue de 833 adolescentes $(47.2 \%$ chicos y $52.8 \%$ chicas) con edades comprendidas entre los 12 y los 17 años de edad ( $M=$ 13.89 y $S D=1.28$ ) escolarizados en 6 centros educativos de enseñanza secundaria obligatoria (ESO) ubicados en la provincia de Alicante (España). Los participantes procedían de centros públicos (53.3\%) y centros concertados (47.7\%).

El promedio de datos faltantes fue de $1.8 \%$ y nunca superior al $4 \%$ para una medida individual. Los datos faltantes para escalas o subescalas fueron procesados utilizando el método de imputación por regresión. Los valores atípicos univariados se detectaron mediante la exploración de puntajes estandarizados. Estos valores fueron aquellos cuyos puntajes estandarizados tuvieron un valor absoluto superior a 4 (Hair et al., 2016). Para la detección multivariante, se calculó la distancia de Mahalanobis. Se identifica un valor atípico multivariado si la probabilidad asociada a una distancia Mahalanobis es 0.001 o menos (Tabachnick y Fidell, 2007).

\subsection{Procedimiento}

Las escuelas fueron seleccionadas en función de su condición, pública o privada. En primer lugar, se contactó con la dirección de los seis centros educativos seleccionados para explicar el proyecto de investigación y solicitar su aceptación, expresándoles que su participación sería anónima, voluntaria y confidencial. Obtenido el permiso de los centros, se solicitó un espacio para impartir un seminario informativo para el profesorado con la finalidad de explicar los objetivos de la investigación y solicitar su colaboración. También se les informó respecto de su importante rol en la investigación al tener que evaluar a cada alumno en las tres dimensiones del cuestionario ajuste escolar. Paralelamente, se remitió a todas las familias, vía el alumnado, una carta en la que se les solicitaba por escrito su consentimiento para que sus hijos/as participasen en el estudio (.8\% de los padres no dieron su consentimiento).

\subsection{Análisis de datos}

Se llevó a cabo una partición de la muestra total en dos submuestras a partir de los casos pares e impares. Primeramente, se realizó un análisis factorial exploratorio con la primera sub-muestra utilizando el paquete estadístico SPSS (versión 20) con objeto 
de analizar la estructura factorial, y se calcularon los análisis de consistencia interna y las propiedades psicométricas de la escala AMAN-A. Seguidamente, se realizaron análisis factoriales confirmatorios con la segunda sub-muestra, utilizando el software EQS 6, que mide el ajuste robusto del modelo a través del TLI, CFI (valores $>.90$ para un buen ajuste) y RMSEA (valores $<.08$ para un buen ajuste; Bentler, 2006), utilizando la estimación de máxima verosimilitud. En cuanto al análisis estadístico, el presente estudio desarrolla diversos procedimientos descriptivos (medidas de tendencia central, de dispersión y de posición), comparaciones significativas (ANOVAS; $p<0,05$ ). Los tamaños del efecto fueron calculados utilizando la $\mathrm{d}$ de Cohen (Cohen, 1988).

\subsection{Instrumento}

Escala de actitudes hacia el medio ambiente natural (AMAN-A).

Análisis previos.

Esta escala se ha elaborado para medir las actitudes hacia el medio ambiente natural en adolescentes. Previamente a los análisis para la validación de la escala se realizaron los pasos recomendados para la construcción de una escala empírica (Muñiz y Fonseca-Pedrero, 2019): 1) se solicitó a 264 adolescentes que escribieran libremente todo lo que se les ocurriera sobre sus actitudes hacia el medio ambiente natural (previamente se les explicó el significado de actitud hacia el medio ambiente natural); 2) se construyeron 72 ítems a partir de esta información; 3) se suprimieron aquellos ítems que tenían una misma connotación semántica, reduciendo la escala a 21 ítems; 4) a través de una evaluación interjueces, se seleccionaron un total de 14 ítems con un nivel de concordancia del 95\%; 5)se realizó una aplicación piloto $(N=228)$ y, tras sucesivos análisis, la escala final quedó constituida por 9 ítems con un rango de respuesta de 1 a 5 (nunca, pocas veces, algunas veces, muchas veces y siempre).

\section{RESULTADOS}

\subsection{Análisis factorial exploratorio}

Análisis factorial exploratorio y fiabilidad. Los resultados de la prueba de Kaiser-Meyer-Olkin (.78) y de la prueba de Bartlett (Chi-cuadrado (36) $=929.187 ; p$ $<.001)$ fueron satisfactorios, indicando la idoneidad de realizar un análisis factorial. El método de factorización utilizado fue el de Componentes Principales con rotación Promax, que permite que los factores estén correlacionados. Una vez obtenidos los resultados del análisis factorial, la selección del número de factores se realizó teniendo en cuenta la regla de Kaiser y el gráfico de sedimentación de Cattell. Así, se observó que había dos factores que mostraban autovalores iniciales superiores 
a 1 (regla de Kaiser) y el gráfico de sedimentación mostraba también una caída importante de la pendiente a partir del segundo factor.

Los dos factores obtenidos explican en conjunto el 53.73\% de la varianza (tabla 1). Analizando el contenido de los ítems que integran cada uno de estos factores, se observa una estructura teóricamente coherente. El primer factor, al que denominamos actitud proximal hacia el medioambiente (APM), explica el 34.49\% de la varianza total e incluye 5 ítems relativos a las actitudes favorables y de respeto hacia el medioambiente. El segundo factor, denominado actitud distal hacia el medio ambiente (ADM), explica el 19.24\% de la varianza total e incluye 4 ítems que hacen referencia a actitudes distantes y con frágil compromiso con el medioambiente. Todos los ítems mostraron saturaciones superiores a .40 e índices de homogeneidad adecuados (tabla 1).

Tabla 1. Medias, desviaciones típicas e índices de homogeneidad de los ítems y análisis factorial exploratorio (primera sub-muestra)

\begin{tabular}{|c|c|c|c|c|c|}
\hline & \multirow[t]{2}{*}{$M$} & \multirow[t]{2}{*}{$D T$} & \multirow[t]{2}{*}{$\begin{array}{l}\text { Índice de } \\
\text { homogeneidad }\end{array}$} & \multicolumn{2}{|c|}{$\begin{array}{l}\text { Factorial } \\
\text { exploratorio }\end{array}$} \\
\hline & & & & F1 & F2 \\
\hline $\begin{array}{l}\text { 1. Para poder sobrevivir necesitamos proteger el } \\
\text { medio ambiente natural. }\end{array}$ & 2.57 & .82 & .56 & .73 & -.04 \\
\hline $\begin{array}{l}\text { 2. La educación en el colegio/instituto sobre el } \\
\text { medio ambiente natural es fundamental para } \\
\text { protegerlo. }\end{array}$ & 3.17 & .87 & .41 & .63 & -.034 \\
\hline $\begin{array}{l}\text { 3. Para vivir mejor hay que destruir el medio } \\
\text { ambiente natural. }\end{array}$ & 1.78 & .88 & .41 & -.26 & .63 \\
\hline $\begin{array}{l}\text { 4. El medio ambiente natural debe ser respetado por } \\
\text { todos. }\end{array}$ & 2.06 & .98 & .61 & .78 & -.16 \\
\hline $\begin{array}{l}\text { 5. La protección del medio ambiente natural es } \\
\text { obligación del gobierno, no mía. }\end{array}$ & 1.38 & .70 & .71 & -.21 & .84 \\
\hline $\begin{array}{l}\text { 6. Si observara a alguien destruyendo el medio } \\
\text { ambiente natural lo denunciaría. }\end{array}$ & 3.04 & 1.00 & .36 & .53 & -.41 \\
\hline $\begin{array}{l}\text { 7. Todos/as deberíamos participar en acciones } \\
\text { encaminadas a proteger y mejorar el medio } \\
\text { ambiente natural (como por ejemplo reciclar). }\end{array}$ & 2.38 & .78 & .66 & .81 & -.29 \\
\hline $\begin{array}{l}\text { 8. Las normas y leyes de protección del medio } \\
\text { ambiente natural no sirven para nada. }\end{array}$ & 1.57 & .83 & .66 & -.12 & .77 \\
\hline $\begin{array}{l}\text { 9. No me importa lo que le ocurra al medio } \\
\text { ambiente natural. }\end{array}$ & 1.31 & .65 & .52 & -.21 & .72 \\
\hline
\end{tabular}

Nota. F1 (Actitud Proximal hacia el Medio Ambiente Natural); F2 (Actitud Distal hacia el Medio Ambiente Natural); M (Media); DT (Desviación Típica). 


\subsection{Análisis factorial confirmatorio}

Seguidamente, se realizaron los análisis factoriales confirmatorios. Se examinó un modelo de dos factores correlacionados para los 9 ítems de la escala utilizando la segunda sub-muestra, con la finalidad de realizar así una validación cruzada de la estructura factorial obtenida (Worthington y Whittaker, 2006). Se utilizó el método de estimación de máxima verosimilitud robusta para todos los análisis, debido a la falta de normalidad multivariada de los datos (coeficiente Mardia - 36.39). En la tabla 2 se observan los coeficientes de asimetría y curtosis de los ítems que integran la escala y se constata que hay una ausencia de normalidad, en la medida en que los coeficientes de ambas medidas de dispersión son superiores \pm 1 . Aunque con el método de máxima verosimilitud se asume el supuesto de la normalidad multivariada, resulta razonablemente robusto a un incumplimiento moderado (Muthén y Kaplan, 1985). Por esta razón, se utilizó la Chicuadrado de Satorra-Bentler $\left(\mathrm{SB} \chi^{2}\right)$, que se recomienda en situaciones de ausencia moderada de normalidad en la distribución de los datos (Curran, West y Finch, 1996).

Por otro lado, se han tenido en cuenta las actuales recomendaciones sobre la conveniencia de utilizar más de una medida de ajuste del modelo. Por esta razón, se han considerado la Chi-cuadrado, el índice de ajuste comparativo (CFI robusto), el índice de ajuste no normado de Bentler-Bonett (NNFI), el índice de ajuste normado (NFI) y el error de aproximación cuadrático medio (RMSEA). Se considera habitualmente como un buen ajuste del modelo, cuando los índices del CFI, NFI y NNFI son superiores a .95 , y aceptables si superan el .90. En el caso del RMSEA se considera un buen ajuste cuando los valores son inferiores a $.05, \mathrm{y}$ un ajuste aceptable con valores entre $.05 \mathrm{y}$ .08 (Browne y Cudeck, 1992). Los índices obtenidos en nuestros análisis fueron los siguientes: $\mathrm{S}-\mathrm{B} \chi^{2}=24.84, g l=20, p>.05$, CFI $=.992, \mathrm{NNFI}=.985, \mathrm{NFI}=.959, \mathrm{RMSEA}=$ $.024(.000-.051)$. Con estos indicadores se puede considerar que el ajuste es aceptable y, por tanto, se confirma la estructura factorial de dos factores (figura 1).

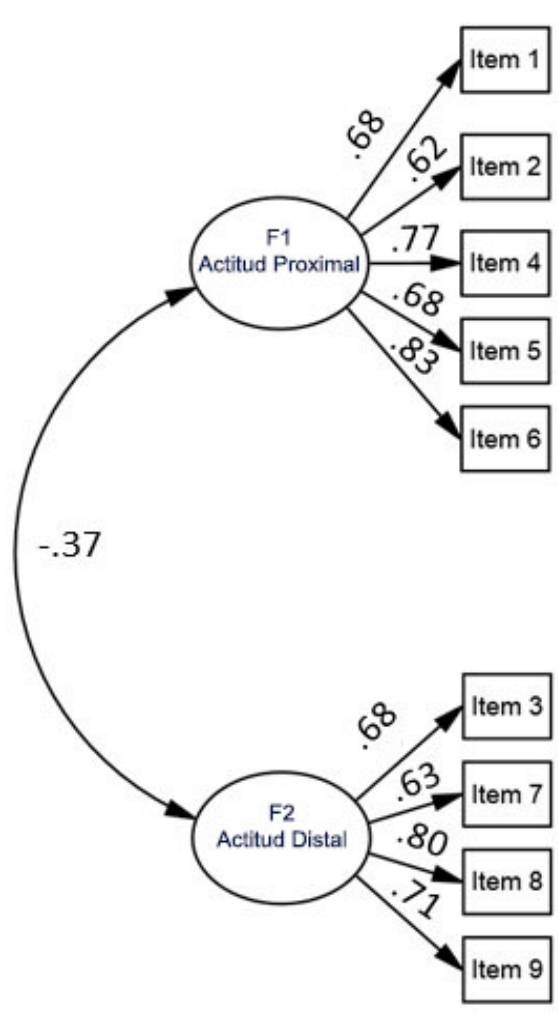

Figura 1. Modelo de medida de la escala AMAN-A. Correlaciones: $r$ $(\mathrm{F} 1-\mathrm{F} 2)=.368$; CFA fit index: [S-Bc2 $=24.84, \mathrm{gl}=20, \mathrm{p}>.05, \mathrm{CFI}=.992$, $\mathrm{NNFI}=.985, \mathrm{NFI}=.959, \mathrm{RMSEA}=$ $.024(.000-.051)]$ 
Tabla 2. Medias, desviaciones típicas, asimetría y curtosis de los ítems y análisis factorial confirmatorio (solución estandarizada) en la segunda sub-muestra.

\begin{tabular}{|c|c|c|c|c|c|c|}
\hline & \multirow[t]{2}{*}{$M$} & \multirow[t]{2}{*}{$D T$} & \multirow[t]{2}{*}{ Asimetría } & \multirow[t]{2}{*}{ Curtosis } & \multicolumn{2}{|c|}{$\begin{array}{c}\text { Factorial } \\
\text { confirmatorio }\end{array}$} \\
\hline & & & & & F1 & F2 \\
\hline $\begin{array}{l}\text { 1. Para poder sobrevivir necesitamos proteger el } \\
\text { medio ambiente natural. }\end{array}$ & 2.57 & .88 & -.003 & -.67 & .68 & \\
\hline $\begin{array}{l}\text { 2. La educación en el colegio/instituto sobre el } \\
\text { medio ambiente natural es fundamental para } \\
\text { protegerlo. }\end{array}$ & 3.09 & .90 & -.61 & -.63 & .62 & \\
\hline $\begin{array}{l}\text { 3. Para vivir mejor hay que destruir el medio } \\
\text { ambiente natural. }\end{array}$ & 1.78 & .88 & 1.06 & .42 & & .68 \\
\hline $\begin{array}{l}\text { 4. El medio ambiente natural debe ser respetado } \\
\text { por todos. }\end{array}$ & 2.09 & 1.06 & .52 & -.99 & .77 & \\
\hline $\begin{array}{l}\text { 5. La protección del medio ambiente natural es } \\
\text { obligación del gobierno, no mía. }\end{array}$ & 1.44 & .81 & 2.06 & 3.46 & & .63 \\
\hline $\begin{array}{l}\text { 6. Si observara a alguien destruyendo el medio } \\
\text { ambiente natural lo denunciaría. }\end{array}$ & 3.00 & 1.04 & -.62 & -.89 & .68 & \\
\hline $\begin{array}{l}\text { 7. Todos/as deberíamos participar en acciones } \\
\text { encaminadas a proteger y mejorar el medio } \\
\text { ambiente natural (como por ejemplo reciclar). }\end{array}$ & 2.46 & .81 & .15 & -.41 & .83 & \\
\hline $\begin{array}{l}\text { 8. Las normas y leyes de protección del medio } \\
\text { ambiente natural no sirven para nada. }\end{array}$ & 1.58 & .86 & 1.55 & 1.57 & & .80 \\
\hline $\begin{array}{l}\text { 9. No me importa lo que le ocurra al medio } \\
\text { ambiente natural. }\end{array}$ & 1.31 & .66 & 2.54 & 6.45 & & .71 \\
\hline
\end{tabular}

Nota. Todos los coeficientes son significativos ( $<.001$ ); F1 (Actitud Proximal hacia el medio ambiente natural); F2 (Actitud Distal hacia el medio ambiente natural); M (Media); DT (Desviación Típica).

Fiabilidad. El análisis de fiabilidad reveló un alfa de Cronbach de .78 y .75 para el factor proximal y el factor distal respectivamente, y para la escala completa fue de .80 . Se calcularon otros índices de fiabilidad como la varianza media extractada que fue de .51 y .50 , (debe ser .50 o mayor) y para la escala completa fue de .51. Por otro lado, la fiabilidad compuesta fue de .79 y .74, y para la escala completa fue de .87. Por último, el omega de Mcdonald fue de .83 y .80 , y para la escala completa fue de .90 .

\subsection{ANOVA}

Primeramente, se realizó un ANOVA entre las dos dimensiones o factores de la escala estudiada para analizar las diferencias de medias en función del sexo. En 
la tabla 3, se expresan las puntuaciones medias, las desviaciones típicas, los valores $F$ y el tamaño del efecto en los grupos de chicos y chicas. Como se aprecia en la tabla, los chicos moraron mayor puntuación en Actitud Distal pero no se observaron diferencias entre los géneros en Actitud Proximal.

Tabla 3. Estadísticos descriptivos, valores $\mathrm{F}$ y tamaño del efecto de las dimensiones de la escala de actitud hacia el medioambiente.

\begin{tabular}{|c|c|c|c|c|}
\hline & Sexo & & $F(1,831)$ & $\eta^{2}$ \\
\hline & Chicos & Chicas & & \\
\hline Actitud Proximal & $2.63(.65)$ & $2.65(.61)$ & .262 & .000 \\
\hline Actitud Distal & $1.61(.63)$ & $1.44(.51)$ & $19.262 * * *$ & .023 \\
\hline
\end{tabular}

\subsection{Validez convergente:}

La Escala de Empatía con el Medio Ambiente Natural (EES) (Musitu-Ferrer et al., 2019a), consta de 11 ítems tipo Likert que miden la empatía con el medio ambiente natural, con un rango de respuesta que oscila entre 1 (nunca) y 5 (siempre). Consta de dos dimensiones: I. Empatía cognitiva hacia el medio ambiente natural -ECM- (por ejemplo, "Procuro ponerme en el lugar de los demás cuando provocan un problema ambiental concreto") $\Omega=.83, \alpha=.83, \mathrm{FC}=.81$, y, II, Empatía emocional - (por ejemplo, "Me siento feliz cuando veo a otras personas disfrutar del medio ambiente sin hacer daño a los seres vivos") $\Omega=.86, \alpha=.79, \mathrm{FC}=.78$. El análisis factorial confirmatorio (CFA) muestra un buen ajuste del modelo a los datos [S-B $\chi^{2}$ $=78.43, g l=43, p<.001, \mathrm{CFI}=.984$, $\mathrm{RMSEA}=.031(.020-.042)]$ con $\mathrm{VME}=.51$.

La Escala de Ajuste Escolar (-PROF-A-) (Cava et al., 2015), está constituida por 13 ítems tipo Likert que miden el ajuste escolar según el profesorado, con un rango de respuesta de 0 (muy bajo/muy malo) a 9 (muy alto/muy bueno), el ajuste escolar según el profesorado. Consta de tres factores: I. Ajuste social, (por ejemplo, "La relación del alumno con sus compañeros/as") $\Omega=.92, \alpha=.91$, FC =.86; II. Competencia académica, (por ejemplo, "El interés y la atención del alumno/a en lo que se hace en clase") $\Omega=.94, \alpha=.93, \mathrm{FC}=.89$; y, III. Implicación familiar, (ejemplo: "El grado de implicación de la familia en el seguimiento escolar del hijo/a") $\Omega=.95, \alpha=.95, \mathrm{FC}=.90$. El análisis factorial confirmatorio (CFA) muestra un buen ajuste del modelo a los datos $\left[\mathrm{S}-\mathrm{B} \chi^{2}=197.79, g l=53, p<.001\right.$, CFI $=.976$, RMSEA $=.057(.049-.066)]$ con $\mathrm{VME}=.77$.

Finalmente, se realizó un análisis correlacional entre los factores de las escalas de actitud y empatía hacia el medioambiente y los de ajuste escolar con ambas submuestras independientemente (tabla 4). Se observaron correlaciones positivas entre 
la actitud proximal y los dos factores de la empatía con el medio ambiente natural: la emocional y la cognitiva, tanto en la primera sub-muestra $(r=.14, r=.22, p<$ $.01)$, como en la segunda $(r=.15, p<.01 ; r=.12, p<.05)$. También se observó una correlación positiva con implicación familiar $(r=.12, p<.05)$ en la primera sub-muestra $\mathrm{y}$, en competencia académica en la segunda sub-muestra $(r=.21, p$ $<.01)$. Respecto de la actitud distal, se obtuvieron correlaciones negativas con la empatía cognitiva hacia el medioambiente, con la competencia académica y con la implicación familiar $(r=-.19, r=-.24, r=-.13, p<.01$, en la primera sub-muestra; $\mathrm{y}$ $r=-.22, r=-.29, r=-.15, p<.01$, en la segunda), así como con la empatía emocional hacia el medioambiente en la segunda sub-muestra $(r=-.22, p<.01)$.

Tabla 4. Correlaciones entre la actitud hacia el medioambiente, empatía hacia el medioambiente y la percepción del alumno/a por el/la profesor/a (primera submuestra en la parte superior de la diagonal)

\begin{tabular}{lccccccc}
\hline \multicolumn{1}{c}{ Variable } & 1 & 2 & 3 & 4 & 5 & 6 & 7 \\
\hline Actitud hacia el medioambiente natural & & & & & & & \\
\hline 5. $\quad$ Actitud Proximal & & $-.31^{* *}$ & $.14^{* *}$ & $.22^{* *}$ & .03 & $.23^{* *}$ & $.12^{*}$ \\
\hline 6. $\quad$ Actitud Distal & $-.35^{* *}$ & & -.09 & $-.19^{* *}$ & -.08 & $-.24^{* *}$ & $-.13^{* *}$ \\
\hline Empatía hacia el medioambiente natural & & & & & & & \\
\hline 7. $\quad \begin{array}{l}\text { Empatía Emocional hacia el } \\
\text { medioambiente natural }\end{array}$ & $.15^{* *}$ & $-.22^{* *}$ & & $.64^{* *}$ & $.16^{* *}$ & $.18^{* *}$ & $.13^{* *}$ \\
\hline $\begin{array}{l}\text { 8. Empatía Cognitiva hacia el } \\
\quad \text { medioambiente natural }\end{array}$ & $.12^{*}$ & $-.22^{* *}$ & $.70^{* *}$ & & $.10^{*}$ & $.20^{* *}$ & $.10^{*}$ \\
\hline Ajuste Escolar & & & & & & & \\
\hline 9. Ajuste Social & .01 & -.06 & $.12^{*}$ & $.12^{*}$ & & $.48^{* *}$ & $.42^{* *}$ \\
\hline 10. Competencia Académica & $.21^{* *}$ & $-.29^{* *}$ & $.12^{*}$ & $.17^{* *}$ & $.48^{* *}$ & & $.55^{* *}$ \\
\hline 7. Implicación Familiar & .05 & $-.15^{* *}$ & .08 & .07 & $.36^{* *}$ & $.51^{* *}$ & \\
\hline
\end{tabular}

Nota: $* p<.05, * * p<.01$.

\section{DISCUSIÓN}

El objetivo de este estudio fue elaborar una escala de actitudes hacia el medio ambiente natural para adolescentes y analizar sus propiedades psicométricas y su estructura factorial. La escala se construyó a partir de los escritos de 264 adolescentes respecto de sus actitudes hacia el medio ambiente en general y se ha validado con una muestra de 833 adolescentes. Esta escala, que presenta unas adecuadas propiedades psicométricas tiene la virtud de llenar el vacío existente en el ámbito de la evaluación de las actitudes hacia el medio ambiente para adolescentes en lengua española. Además, tiene la peculiaridad, a nuestro juicio muy significativa, de que 
se ha elaborado a partir de las opiniones expresadas por los adolescentes respecto del medio ambiente natural. Como ya ha quedado constatado, son numerosas las escalas para la evaluación de las actitudes hacia el medio ambiente natural que se han construido en estos últimos treinta años, pero no tenemos constancia de escalas construidas desde y para adolescentes. Además, esta escala tiene la virtud de la brevedad, una característica importante cuando se trata de investigar en el ámbito de la Educación Ambiental en este periodo evolutivo.

El modelo que surge de los análisis realizados está constituido por dos factores, que muestran una alta coherencia teórica. El primer factor, denominado Actitud Proximal hace referencia a la actitud de compromiso, respeto ("El medio ambiente natural debe ser respetado por todos") y de identidad con el medio ambiente natural con alusiones a la intención conductual como, por ejemplo, ("todos/as deberíamos participar en acciones encaminadas a proteger y mejorar el medio ambiente natural, como por ejemplo reciclar"). El segundo factor, denominado Actitud Distal, evalúa la lejanía, la falta de identidad y la indiferencia hacia el medio ambiente natural como por ejemplo, ("no me importa lo que le ocurra al medio ambiente natural").

Además de la coherencia en su estructura y su interés teórico, la escala AMAN-A, presenta unos adecuados índices de fiabilidad y validez convergente. Se ha observado que se relaciona con la empatía emocional y cognitiva hacia el medioambiente, con la competencia académica, e igualmente se correlaciona con la implicación familiar, aunque débilmente. En el caso de la actitud distal se correlaciona negativamente con la empatía cognitiva y emocional hacia el medioambiente, con la competencia académica y con la implicación familiar. Estas relaciones son relevantes porque dotan de contenido y significado a la escala AMAN-A. También es muy sugerente la relación de las actitudes proximales y negativa de las actitudes distales con la competencia académica que se recoge en AMAN-A, que es un factor del ajuste escolar, en primer lugar porque son pocos los trabajos en los que se analicen conjuntamente estas dimensiones, aunque sí se han relacionado de forma independiente con la empatía y la conectividad con el medio ambiente (PavalacheIlie y Cazan, 2018; Xie, Bagozzi y Grønhaug, 2019), en segundo lugar, porque se integra en el ámbito de la educación ambiental, el ajuste escolar y en su dimensión académica (Musitu-Ferrer, León-Moreno, y Callejas Jerónimo, 2019c).

En este sentido, son numerosos los trabajos en los que en estos últimos años se ha observado la importancia de las Aulas de la Naturaleza en los centros escolares porque, por una parte, promueven actitudes proambientales junto con la empatía y la conectividad y, por otra, porque se ha constatado de manera consistente que la vivencia del alumnado en estas aulas mejora el rendimiento académico en general (Musitu-Ferrer et al., 2019b); la motivación intrínseca (Hobbs, 2015); la conectividad con la escuela (Becker, Lauterbach, Spengler, Dettweiler y Mess, 2017; Kuo, Barnes y Jordan, 2019); el estado de ánimo (Takayama et al., 2014); las actitudes hacia el medio ambiente (Biasutti y Frate, 2017) y la conducta proambiental (Amérigo, García 
y Sánchez, 2013). De ahí la importancia de la escala AMAN-A y sus relaciones con el ajuste escolar y la empatía.

También, se han observado diferencias en función del género en el sentido de que las chicas muestran una menor actitud distal que los chicos, aunque no difieren de estos respecto de la actitud proximal. Este resultado nos remite a la idea de que, aunque chicos y chicas no difieren en la actitud proximal, en la actitud distal los chicos se sienten más lejanos y menos comprometidos con el medio ambiente que las chicas, es decir, parece que ellos tienen sentimientos más contradictorios. Hay dos teorías desde las que se podrían explicar estas diferencias: la primera alude a la teoría de las oportunidades -es la que goza de menos apoyo-, que sugiere que los hombres y las mujeres no necesariamente tienen motivaciones diferentes y que las diferencias de género se pueden explicar por las diferencias en las constricciones externas y por las oportunidades que gozan hombres y mujeres; $y$, la segunda, alude a las diferentes realidades tanto biológicas, como psicológicas y experienciales las cuales implican un diferente enfoque de los problemas y de las realidades sociales (Gottfredson y Hirschi, 1990; Torgler, Garcia-Valiñas y Macintyre, 2008).

En esta última se encuentran la teoría de la socialización de género y las teorías de los roles de género a las que se acude con frecuencia para explicar las diferencias de género observadas en el ambientalismo (Dietz, Kalof y Stern, 2002; Xiao y McCright, 2015; Zelezny, Chua y Aldrich, 2000). Las mujeres suelen estar socializadas para empatizar con las necesidades y el bienestar de otras personas y para ser más interdependientes y cooperativas. Esta mayor empatía adquirida por ellas durante la socialización y las expectativas y experiencias del rol de género, dan lugar a una mayor preocupación hacia los animales y el entorno natural (Milfont y Sibley, 2016; Musitu Ferrer et al., 2019b).

Finalmente, el instrumento analizado en este estudio, AMAN-A, muestra una estructura bidimensional de las actitudes hacia el medio ambiente en adolescentes; la primera representa la proximidad e identidad con el medio ambiente y, la segunda, la lejanía y la ausencia de compromiso. La escala presenta unas adecuadas propiedades psicométricas y creemos que puede contribuir a enriquecer la investigación de las actitudes hacia el medio ambiente en la adolescencia. Además, es un instrumento que permitirá al profesorado conocer de manera puntual las actitudes de su alumnado hacia el medio ambiente. Como subrayan Meinhold y Malkus (2005), las actitudes que los adolescentes tienen sobre el medio ambiente influirán en las decisiones futuras sobre los recursos naturales, y muy importante, cómo se respetará, utilizará y cuidará.

\section{REFERENCIAS}

Ajzen, Icek y Madden, Thomas (1986). Prediction of goal-directed behavior: Attitudes, intentions, and perceived behavioral control. Journal of Experimental Social Psychology, 22, pp. 453-474. 
Fiabilidad y validez de la escala de actitudes... - D. Musitu-Ferrer y otros

Akehurst, Gary; Afonso, Carolina y Gonçalves, Helena (2012). Re-examining green purchase behaviour and the green consumer profile: New evidences. Management Decision, 50(5), pp. 972-988. https://doi.org/10.1108/00251741211227726

Allport, Gordon W. (1968). La personalidad. Su configuración y desarrollo. Barcelona: Herder.

Álvarez, P; Inmaculada de la Fuente, Helena; García, Juan y Fernández, $M^{a}$ José. ( $\uparrow$. १ $)$. Evaluación de las actitudes ambientales en la ESO: análisis de un instrumento. Alambique: Didáctica de las ciencias experimentales, (22), 77-86.

Amador Muñoz, Luis V. y Esteban Ibáñez, Macarena (2011). Desde la educación social a la educación ambiental. Hacía una intervención educativa socioambiental. Revista de Humanidades, n. 18, pp. 147-160. DOI: https://doi.org/10.5944/rdh.18.2011.12885

Amérigo, María; García, Juan y Sánchez, Trinidad (2013). Actitudes y comportamiento hacia el medio ambiente natural. Salud medioambiental y bienestar emocional. Universitas Psychologica, 12(3), pp. 845-856. https://doi.org/10.11144/Javeriana. UPSY12-3.acma

Ariza, Carla (2017). Environmental education as global strategy for sustainability. Revista Boletín Redipe, 6(5), pp. 64-70.

Bamberg, Sebastian y Moser, Guido (2007). Twenty years after Hines, Hungerford, and Tomera: A new meta-analysis of psycho-social determinants of pro-environmental behaviour. Journal of Environmental Psychology, 27, pp. 14-25. https://doi. org/10.1016/j.jenvp.2006.12.002

Becker, Christoph; Lauterbach, Gabriele; Spengler, Sarah; Dettweiler, Ulrich y Mess, Filip (2017). Effects of regular classes in outdoor education settings: A systematic review on students' learning, social and health dimensions. International Journal of Environmental Research and Public Health, 14(5), pp. 1-21. https://doi.org/10.3390/ ijerph14050485

Bentler, Peter. M. (2006). EQS 6 structural equations program manual. University of Encino: California.

Bergman, Brenda Gail (2016). Assessing impacts of locally designed environmental education projects on students' environmental attitudes, awareness, and intention to act. Environmental Education Research, 22(4), pp. 480-503. https://doi.org/10.1080 113504622.2014 .999225

Biasutti, Michele y Frate, Sara (2017). A validity and reliability study of the Attitudes toward Sustainable Development scale. Environmental Education Research, 23(2), pp. 214-230. https://doi.org/10.1080/13504622.2016.1146660

Biasutti, Michele y Surian, Alessio (2012). The Students' Survey of Education for Sustainable Development Competencies: A comparison among faculties. Discourse and Communication for Sustainable Education, 3(1), pp. 75-82. https://doi. org/10.2478/v10230-012-0005-y

Bogner, Franz X. y Wiseman, Michael (2006). Adolescents' attitudes towards nature and environment: Quantifying the 2-MEV model. Environmentalist, 26(4), pp. 247-254. https://doi.org/10.1007/s10669-006-8660-9 
Brody, Samuel D.; Highfield, Wes y Alston, Leticia (2004). Does location matter? Measuring Environmental Perceptions of Creeks in Two San Antonio Watersheds. Environment and Be, 36(2), pp. 229-251. https://doi.org/10.1177/0013916503256900

Browne, Michael W. y Robert Cudeck (1992). Alternative ways of assessing model fit. Sociological Methods \& Research,21(2), pp. 230-258. https://doi. org/10.1177/0049124192021002005

Castedano, Celedonio (1995). Escala de las conductas pro-ambientales (EAPA) en alumnos universitarios. Revista Complutense de Investigación, 6(2).

Cava, María-Jesús; Povedano, Amapola; Buelga, Sofia y Musitu, Gonzalo (2015). Análisis psicométrico de la Escala de Ajuste Escolar Percibido por el Profesor (PROF-A). Psychosocial Intervention, 24(2), pp. 63-69. https://doi.org/10.1016/j. psi.2015.04.001

Christensen, Rhonda y Knezek, Gerald (2015). The climate change attitude survey: Measuring middle school student beliefs and intentions to enact positive environmental change. International Journal of Environmental and Science Education, 10(5), pp. 773-788. https://doi.org/10.12973/ijese.2015.276a

Cohen, Jacob (1988). Statistical power analysis for the behavioural sciences (2nd ed.). Hillsdale, NJ: Erlbaum.

Corral-Verdugo, Víctor; Frijo, Blanca y Tapia, César (2004). Propensiones psicológicas en niños de sexto grado de primaria. Validez de un instrumento. Anuario de Investigaciones Educativas, 7, pp. 31-44.

Curran, Patrick J.; West, Stephen G. y Finch, John F. (1996). The robustness of test statistics to nonnormality and specification error in confirmatory factor analysis. Psychological Methods, 1(1), pp. 16-29. https://doi.org/10.1037/1082-989X.1.1.16

Dietz, Thomas; Kalof, Linda y Stern, Paul (2002). Gender, values, and environmentalism. Social Science Quarterly, 83(1), pp. 353-364. https://doi.org/10.1111/1540$\underline{6237.00088}$

Duarte, Rosa; Escario, José-Julián y Sanagustín, María-Victoria (2017). The influence of the family, the school, and the group on the environmental attitudes of European students. Environmental Education Research, 23(1), pp. 23-42. https://doi.org/10.10 $\underline{80 / 13504622.2015 .1074660}$

Dunlap, Riley E.; Van Liere, Kent D.; Mertig, Angela G. y Jones, Robert Emmet (2000). New Trends in Measuring Environmental Attitudes: Measuring Endorsement of the New Ecological Paradigm: A Revised NEP Scale. Journal of Social Issues, 56(3), pp. 425-442. https://doi.org/10.1111/0022-4537.00176

Dunlap, Riley; Gallup, George y Gallup, Alec (1993). Of global concern: Results of the health of the planet survey. Environment: Science and Policy for Sustainable Development, 35(9), pp. 7-15.

Eagly, A. H., y Chaiken, S. (1993). The psychology of attitudes (Harcourt B). Fort Worth, TX.

Escalante, Eduardo; Repetto, Ana María y Mattinello, Gabriela (2012). Exploración y análisis de la actitud hacia la estadística en alumnos de psicología. LIBERABIT, 18(1), pp. 15-26. 
Esteban Ibáñez, Macarena; Amador Muñoz, Luis Vicente y Mateos Claros, Francisco. (2017). Attitudes of university students towards the environment: Environmental education and innovation. Revista de Humanidades, 31, pp. 17-38.

Evans, Gary W.; Brauchle, Gernot; Haq, Aliya; Stecker, Rachel; Wong, Kimberly Wong y Shapiro, Elan (2017). Young children's environmental attitudes and behaviors. Environment and Behavior, 39(5), pp. 635-659. https://doi. org/10.1177/0013916506294252

Fielding, Kelly S.; Terry, Deborah J.; Masser, Barbara M.; y Hogg, Michael A. (2008). Integrating social identity theory and the theory of planned behaviour to explain decisions to engage in sustainable agricultural practices. British Journal of Social Psychology, 47, pp. 23-48. https://doi.org/10.1348/014466607X206792

Fishbein, Martin y Ajzen, Icek (1977). Belief, attitude, intention, and behavior: An introduction to theory and research. Reading, MA: Addison-Wesley.

Fishbein, Martin y Ajzen, Icek (1980). Predicting and understanding consumer behavior: Attitude- behavior correspondence. En Ajzen, Icek y Fishbein, Martin (Eds.). Understanding attitudes and predicting social behavior, pp. 95-125.

Fleury-Bahi, Ghozlane; Marcouyeux, Aurore; Renard, Elise y Roussiau, Nicolas (2015). Factorial structure of the New Ecological Paradigm scale in two French samples. Environmental Education Research, 21(6), pp. 821-831. https://doi.org/10.1080/13 504622.2014.913127

Foster, Richard H. y Mcbeth, Mark K. (1996). Urban-rural influences in U. S. environmental and economic development policy. Jorunal of Rural Studies, 12(4), pp. 387-397.

Fraijo, Blanca; Corral, Víctor; Tapia, Cesar y García, Fernanda. (2012). Adaptación y prueba de una escala de orientación hacia la sustentabilidad en niños de sexto año de educación básica. Revista mexicana de investigación educativa, 17(55), 1091-1117.

Fraijo, Blanca; Tapia, César y Corral, Víctor (2007). Orientación hacia la sustentabilidad en estudiantes universitarios un estudio diagnóstico. En González, Daniel y Maytorena María de los Ángeles (Eds.). Estudios empíricos en educación superior, México: Unison-Conacyt.

Galli, Francielli; de Campos, Camila; Bedim, Livia. M y Sarriera, Jorge (2013). Actitudes hacia el medio ambiente en la infancia: un análisis de niños del sur de Brasil. Revista Latinoamericana de Psicología, 45(3), 461-473.

Gottfredson, Michael R. y Hirschi, Travis (1990). A general theory of crime. Stanford, CA: Stanford University Press.

Hair, Joseph F; Hult, G. Tomas; Ringle, Christian M. y Sarstedt, Marko (2016). A Primer on Partial Least Squares Structural Equation Modelling (PLS-SEM). Thousand Oaks, CA: Sage Publications

Harris, Paul (2006). Environmental perspectives and behavior in China: Synopsis and bibliography. Environment and Behavior, 38(1), pp. 5-21. https://doi. org $/ 10.1177 / 0013916505280087$

Heberlein, Thomas (2012). Navigating Environmental Attitudes. New York: Oxford University Press. 
Heyl, Marianne; Díaz, Emilio Moyano y Cifuentes, Luis (2013). Actitudes y comportamientos ambientales de estudiantes universitarios: Estudio de una universidad chilena. Revista Latinoamericana de Psicologia, 45(3), pp. 487-505. https://doi.org/10.14349/rlp.v45i3.1489

Hobbs, Laura Kate (2015). Play-based science learning activities: Engaging adults and children with informal science learning for preschoolers. Science Communication, 37(3), pp. 405-414. https://doi.org/10.1177/1075547015574017

Holahan, Charles J. (1982). Environmental psychology. New York: Ramdom House.

Kaiser, Florian (1998). A general measure of ecological behavior 1. Journal of Applied Social Psychology, 28(5), pp. 395-422. https://doi.org/10.1111/j.1559-1816.1998. tb01712.x

Katz, Daniel y Scotland, Ezra (1959). A preliminary statement to a theory of attitude structure and change. En Koch, Sigmund (Ed.). Psychology: A study of a science, pp. 423-475. New York: McGraw-Hill.

Kesícíoğlu, Ogur Sedar y Alísínanoğlu, Fatma (2009). The analysis of children's attitude against the environment in the views of various variables. Ahi EvranÜnv. Kırşehir Ĕgitim Fakültesi Dergisi (KEFAD), 10(3), pp. 37-48.

Kollumuss,AnjayAgyeman, Julian(2002). Mind the gap: Why dopeopleactenvironmentally and what are the barriers to pro-environmental behavior? Environmental Education Research, 8 (3) pp. 239-260. https://doi.org/10.1080/13504620220145401

Kotler, Philip; Kartajava, Hermawan y Setiawan, Iwan (2011). Marketing 3.0. Editorial Empresarial.

Kroufek, Roman; Janovec, Jan; Chytrý, Vlastimil y Simonová, Veronica (2016). Environmental attitudes of pre-school children and their parents. En International Technology, Education and Development Conference. Valencia: España.

Kuo, Ming; Barnes, Michael y Jordan, Catherine (2019). Do experiences with nature promote learning? Converging evidence of a cause-and-effect relationship. Frontiers in Psychology, 10(305), pp. 1-9. https://doi.org/10.3389/fpsyg.2019.00305

Lamberth, John (1980). Psicología Social. Madrid: Pirámide.

Leeming, Frank C.; Dwyer, William O. y Bracken, Bruce A. (1995). Children's Environmental Attitude and Knowledge Scale: Construction and Validation. Journal of Environmental Education, 26(3), pp. 22-31. https://doi.org/10.1080/00958964.19 $\underline{95.9941442}$

Levine Debra S. y Strube, Michael J. (2012). Environmental attitudes, knowledge, intentions and behaviors among college students. The Journal of Social Psychology, 152(3), pp. 308-326. https://doi.org/10.1080/00224545.2011.604363

Makki, Maha Haidar; Abd-el-khalick, Fouad y Boujaoude, Saouma (2003). Lebanese Secondary School students' environmental knowledge and attitudes. Environmental Education Research ISSN, 9(1), pp. 21-33. https://doi.org/10.1080/13504620303468

Meinhold, Jana L. y Malkus, Amy J. (2005). Adolescent environmental behaviors: Can knowledge, attitudes and self-efficacy make a difference? Environment and Behavior, 37(4), 511-532. https://doi.org/10.1177/0013916504269665 
Melero, Luis y Buz, José(2002). ¿Existen realmente los estereotipos en los adolescentes hacia los adultos, los mayores y los muy mayores? Imserso, Memoria Técnica, no publicada.

Michalos, Alex C.; Creech, Heather; Swayze, Natalie; Kahlke, P. Maurin; Buckler, Carolee y Rempel, Karen (2012). Measuring knowledge, attitudes and behaviours concerning sustainable development among tenth grade students in Manitoba. Social Indicators Research, 106, pp. 213-238. https://doi.org/10.1007/s11205-011-9809-6

Miguens, María; Álvarez, Paula; González, Encarnación y García, María (2015). Medidas del comportamiento ecológico y antecedentes: Conceptualización y validación empírica de escalas. Universitas Psychologica, 14(1), pp. 15-30. https://doi. org/10.11144/Javeriana.upsy14-1.mcea

Milfont, Taciano L. y Duckitt, John (2004). The structure of environmental attitudes: A first- and second-order confirmatory factor analysis. Journal of Environmental Psychology, 24, 289-303. https://doi.org/10.1016/j.jenvp.2004.09.001

Milfont, Taciano L. y Duckitt, John (2010). The environmental attitudes inventory: A valid and reliable measure to assess the structure of environmental attitudes. Journal of Environmental Psychology, 30(1), pp. 80-94. https://doi.org/10.1016/j. jenvp.2009.09.001

Milfont, Taciano L. y Sibley, Chris G. (2016). Empathic and social dominance orientations help explain gender differences in environmentalism: A one-year Bayesian mediation analysis. Personality and Individual Differences, 90, pp. 85-88. https://doi. org/10.1016/j.paid.2015.10.044

Moreno, Marta; Corraliza, José Antonio y Ruiz, Juan Pedro (2005). Escala de Attitudes Ambientales Hacia Problemas Específicos. Psicothema, 17(3), pp. 502-508.

Moyano-Díaz, Emilio; Cornejo, Felipe A. y Gallardo, Ismael (2011). Creencias y conductas ambientales, liberalismo económico y felicidad. Acta Colombiana de Psicología, 14(2), pp. 69-77.

Muñiz, José y Fonseca-Pedrero, Eduardo (2019). Diez pasos para la construcción de un test. Psicothema, 31(1), pp. 7-16. https://doi.org/10.7334/psicothema2018.291

Musitu-Ferrer, Daniel; Esteban-Ibáñez, Macarena; León-Moreno, Celeste y García, Oscar F. (2019a). Is school adjustment related to environmental empathy and connectedness to nature? Psychosocial Intervention, 28(2), pp. 101-110. https://doi.org/10.5093/ pi2019a8

Musitu-Ferrer, Daniel; León-Moreno, Celeste y Callejas Jerónimo, Juan Evaristo; EstebanIbañez, Macarena; Musitu-Ochoa, Gonzalo (2019b). Relationships between Parental Socialization Styles, Empathy and Connectedness with Nature: Their Implications in Environmentalism. International Journal of Environmental Rresearch and Public Health, 16 (14) 2461. https://doi.org10.3390/ijerph16142461

Musitu-Ferrer, Daniel; León-Moreno, Celeste y Callejas Jerónimo, Juan Evaristo (2019c). Un análisis socioeducativo de la Educación Ambiental y del Aula Natura. Revista de Educación Social, 28, pp. 59-78.

Muthén, Bengt O. y Kaplan, David (1985). A comparison of some methodologies for the factor analysis of non-normal likert variables. British Journal of Mathematical and Statistical Psychology, 38, pp. 171-189. 
Novo, María (2009). La educación ambiental, una genuina educación para el desarrollo sostenible. Revista de educación, n. extraordinario 2009, pp. 195-217.

Ogunbode, Charles A. (2013). The NEP scale: Measuring ecological attitudes/worldviews in an African context. Environment, Development and Sustainability, 15(6), pp. 1477-1494. https://doi.org/10.1007/s10668-013-9446-0

Olsson, Daniel; Gericke, Niklas y Rundgren, S.N. Chang, (2016). The effect of implementation of education for sustainable development in Swedish compulsory schools - assessing pupils' sustainability consciousness. Environmental Education Research, 22(2), pp. 176-202. https://doi.org/10.1080/13504622.2015.1005057

Pavalache-Ilie, Mariela y Cazan, Ana-Maria (2018). Personality correlates of proenvironmental attitudes. International Journal of Environmental Health Research, 28(1), pp. 71-78. https://doi.org/10.1080/09603123.2018.1429576

Pérez Serrano, Gloria (2002). Origen y evolución de la Pedagogía Social. Pedagogía Social. Revista Interuniversitaria, 9, pp. 193-231.

Powell, Robert Baxter; Stern, Marc J.; Krohn, Brian D.; y Ardoin, Nicole (2011). Development and validation of scales to measure environmental responsibility, character development, and attitudes toward school. Environmental Education Research, 17(1), pp. 91-111. https://doi.org/10.1080/13504621003692891

Raudsepp, Maaris (2001). Some socio-demographic and socio-psychological predictors of environmentalism. Trames, 5(55/50), pp. 355-367.

Rosenberg, Milton J. y Hovland, Carl I. (1960) Cognitive, afective and behavioral components of attitudes. En Hovland, Carl I. y Rosenberg, Milton J. (eds), Attitude organization and change, New Haven: Yale University Press.

Schneller, Andrew; Johnson, Bruce y Bogner, Franz (2015). Measuring children's environmental attitudes and values in northwest Mexico: validating a modified version of measures to test the Model of Ecological Values (2-MEV). Environmental Education Research, 21(1), pp. 61-75. https://doi.org/10.1080/13504622.2013.8436 $\underline{48}$

Schultz, Wesley (2000). Empathizing with nature: The effects of perspective taking on concern for environmental issues. Journal of Social Issues, 56, pp. 391-406.

Shephard, Kerry; Harraway, John; Jowett, Tim; Lovelock, Brent; Skeaff, Sheila; Slooten, Liz; Strack, Mick y Furnari, Mary (2014). Longitudinal analysis of the environmental attitudes of university students. Environmental Education Research, 21(6), pp. 1-16. https://doi.org/10.1080/13504622.2014.913126

Soydan, Sema y Samur, Öztürk Samur (2014). A comparative study of the environmental attitudes of 60-66- month-old children and their mothers. Mevlana International Journal of Education 4(2), pp. 27- 36.

Stevenson, Kathryn T.; Peterson, M. Nils y Bondell, Howard D. (2016). The influence of personal beliefs, friends, and family in building climate change concern among adolescents. Environmental Education Research, pp. 1-14. https://doi.org/10.1080/ $\underline{13504622.2016 .1177712}$

Tabachnick, Barbara G. y Fidell, Linda S. (2007). Using Multivariate Statistics (5th ed). Boston, MA: Allyn and Bacon. 
Takayama, Norimasa; Korpela, Kalevi; Lee, Juyoung; Morikawa, Takeshi; Tsunetsugu, Yuko; Park, Bum Jin; Li, Qing; Tyrväinen, Liisa; Miyazaki, Yoshifumi y Kagawa, Takahide (2014). Emotional, restorative and vitalizing effects of forest and urban environments at four sites in Japan. International Journal of Environmental Research and Public Health, 11(7), pp. 7207-7230. https://doi.org/10.3390/ijerph110707207

Taylor, Shirley y Todd, Peter (1995). An integrated model of waste management behavior. A test of household recycling and composting intentions. Environment and Behavior, 27(5), 603-630.

Tilbury, Daniella (2012). Learning to connect: reflections along a personal journey of education and learning for a sustainable future in the context of Rio + 20. Journal of Education for Sustainable Development, 6(1), pp. 59-62.

Torgler, Benno; Garcia-Valiñas, Maria-Angeles y Macintyre, Alison (2008). Differences in preferences towards the environment: The impact of a gender, age and parental effect. FEEM Working Paper, 18.

Uzun, Naim; Gilbertson, Kenneth L.; Keles, Ozgul y Ratinen, Ilkka (2019). Environmental Attitude Scale for Secondary School, High School and Undergraduate Students: Validity and Reliability Study. Journal of Education in Science, Environment and Health, 5(1), 79-90.

Vargas, Catalina; Moreno, Juana; Vázquez, Laura y Gutiérrez, Gustavo (2011). Actitudes ambientales en los estudiantes de nivel superior en México. Luna Azul, (33), pp. 31-36.

Vega, Miguel Ángel; Ferra, Miguel y Quijano Rocio (2009). Valoración del cambio de actitudes hacia el medio ambiente producido por el programa didáctico "EICEA" en los alumnos de Educación Secundaria Obligatoria (14-16 años). Revista electronica de Enseñanza de las Ciencias, 8(3), 1019-1036.

Weigel, Russell y Weigel, Joan (1978). Environmental concern: The development of a measure. Environment and Behavior, 10(1).

Worthington, Roger L. y Whittaker, Tiffany A. (2006). Scale development research: A content analysis and recommendations for best practices. The Counseling Psychologist, 34(6), pp. 806-838. https://doi.org/10.1177/0011000006288127

Xiao, Chenyang y McCright, Aaron M. (2015). Gender Differences in Environmental Concern: Revisiting the Institutional Trust Hypothesis in the USA. Environment and Behavior, 47(1), pp. 17-37. https://doi.org/10.1177/0013916513491571

Xie, Chenyang; Bagozzi, Richard P. y Grønhaug, Kjell (2019). The impact of corporate social responsibility on consumer brand advocacy: The role of moral emotions, attitudes, and individual differences. Journal of Business Research, 95, pp. 514-530. https://doi.org/10.1016/j.jbusres.2018.07.043

Zelezny, Lynnette C.; Chua, Poh-Pheng y Aldrich, Christina (2000). New ways of thinking about environmentalism: Elaborating on gender differences in environmentalism. Journal of Social Issues, 56(3), pp. 443-457. https://doi.org/10.1111/00224537.00177 